\title{
Éducation muséale en ligne et engagement : La participation des musées à l'éducation à l'environnement
}

\section{Cédric Boudjema}

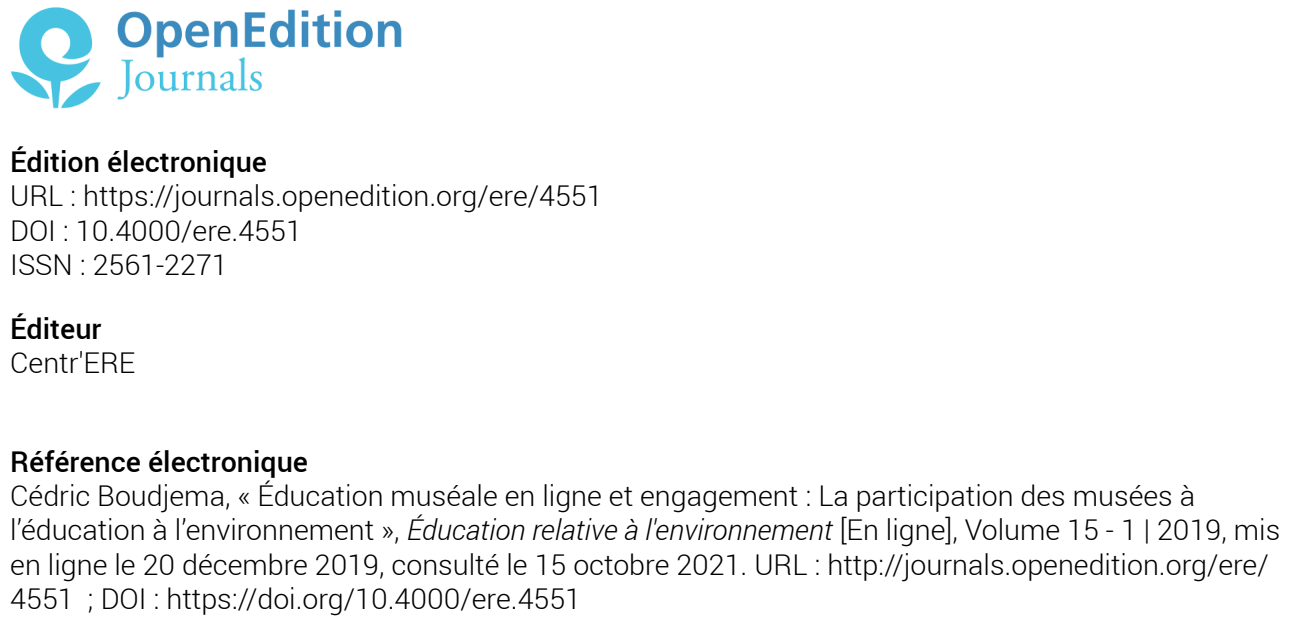

Ce document a été généré automatiquement le 15 octobre 2021

\section{(c) (1) ()}

La revue Éducation relative à l'environnement est mise à disposition selon les termes de la Licence Creative Commons Attribution - Pas d'Utilisation Commerciale 4.0 International. 


\title{
Éducation muséale en ligne et engagement : La participation des musées à l'éducation à l'environnement
}

\author{
Cédric Boudjema
}

1 Le musée peut être un acteur privilégié de l'éducation relative à l'environnement (ERE) puisqu'une des missions essentielles est de faire le lien entre science, patrimoine et société. Les visiteurs peuvent y vivre une expérience sociale. Le musée propose traditionnellement un espace social dans le champ de la vulgarisation c'est-à-dire d'une transformation du contenu scientifique source à un contenu dit cible pour le public et qui nécessite une interprétation des contenus (Davallon, 1999). Mais au-delà de la vulgarisation, dans le cas de l'ERE, une finalité éducative pourrait aller au-delà de la transmission de savoirs : la modification des comportements individuels et collectifs. Notre étude porte sur cette forme de la muséologie de l'environnement via une double médiation: scientifique (vulgarisation) et politique (éducation à la citoyenneté). Il s'agira d'évaluer comment les sites internet instaurent en ligne leur fonction éducative en ERE. Cet article présente les résultats d'une analyse de contenu de sites Web de musées en matière d'ERE et tentera de répondre aux questions suivantes : comment les musées l'abordent-ils et quel est leur engagement pédagogique en la matière sur le Web?

\section{Cadre pour l'analyse en ERE de huit sites web muséaux}

2 La muséologie de l'environnement «s'inscrit dans les trois missions traditionnellement dévolues aux musées de sciences: elle élabore une politique de conservation d'écosystèmes, de paysages, de patrimoine naturel et culturel; elle développe des recherches abordant certaines réalités environnementales; elle instaure des lieux 
éducatifs et culturels qui expriment un discours scientifique relatif à ces réalités. » (Fortin-Debart, 2004, p. 38). Cependant, elle amène le musée à revoir ses fonctions traditionnelles : une mission de conservation non plus d'un patrimoine du passé, mais d'un patrimoine du futur et une mission de médiation qui implique et responsabilise le visiteur face aux enjeux politiques et sociaux. Si la question de l'environnement bouscule les missions traditionnelles du musée, les technologies du numérique vont lui donner les moyens d'en retracer les limites dans la mesure où elle autorise à dépasser la relation à l'objet.

3 Le Web muséal est peu étudié et pourtant les sites Internet condensent de nouvelles missions des musées selon des pédagogies stratégies et intentionnalités pédagogiques propres (Boudjema, 2019). En effet, l'objet muséal comprend désormais des ressources réelles et virtuelles (Paquin et Lemay-Perreault, 2015). Le site Web établit des connexions entre diverses ressources éducatives donnant à l'internaute la possibilité de construire son parcours d'apprentissage (Boudjema, 2016). L'internaute interprète les ressources en ligne selon diverses activités cognitives (Baujard, 2012). Il développe ses propres stratégies d'accès à l'information avec des clés d'accès facilement identifiables. Mais les médiations sémiotiques jouent un rôle structurant dans la production et l'échange des savoirs. Le dispositif technique contribue à la configuration du texte, à son sens et à sa signification. La production médiatique induit une intention et l'internaute est placé devant une monstration d'éléments agencés à l'écran selon une logique communicationnelle. Le concepteur établit une stratégie pour guider la production de sens. Les signes sont répartis dans un espace symbolique (l'écran). La lecture à l'écran est une invitation à agir (adresses faites à l'internaute, l'usage de l'impératif, questions posées à l'internaute, etc.). Le site Web produit du sens qui repose sur un guidage, sur l'implication, l'interaction de l'internaute avec le dispositif en ligne. L'émergence $d u$ sens se base notamment sur un phénomène de reconnaissance culturelle (Jeanneret et Labelle, 2004). Ainsi les sites Web des musées sont des acteurs de contenus réticulaires, éducatifs, mis en scène, ciblés renforçant leur rôle social (Boudjema, 2019) notamment pour l'ERE.

4 Les travaux de Paquin et Lemay-Perreault (2015) ont défini un modèle d'utilisation du musée et du musée virtuel à des fins éducatives en adaptant la situation pédagogique de Legendre (1993). Les autrices ont dégagé quatre types d'interrelations entre les composantes du SOMA (Sujet-Objet-Milieu et Agent) renommées: observationexpérimentation-appropriation (entre le sujet et l'objet), animation-médiationparticipation (entre l'agent et le sujet), conception-mise en œuvre-interprétation (entre l'agent et l'objet) et pédagogie ou andragogie muséale (au centre de toutes les relations) (figure 1). 


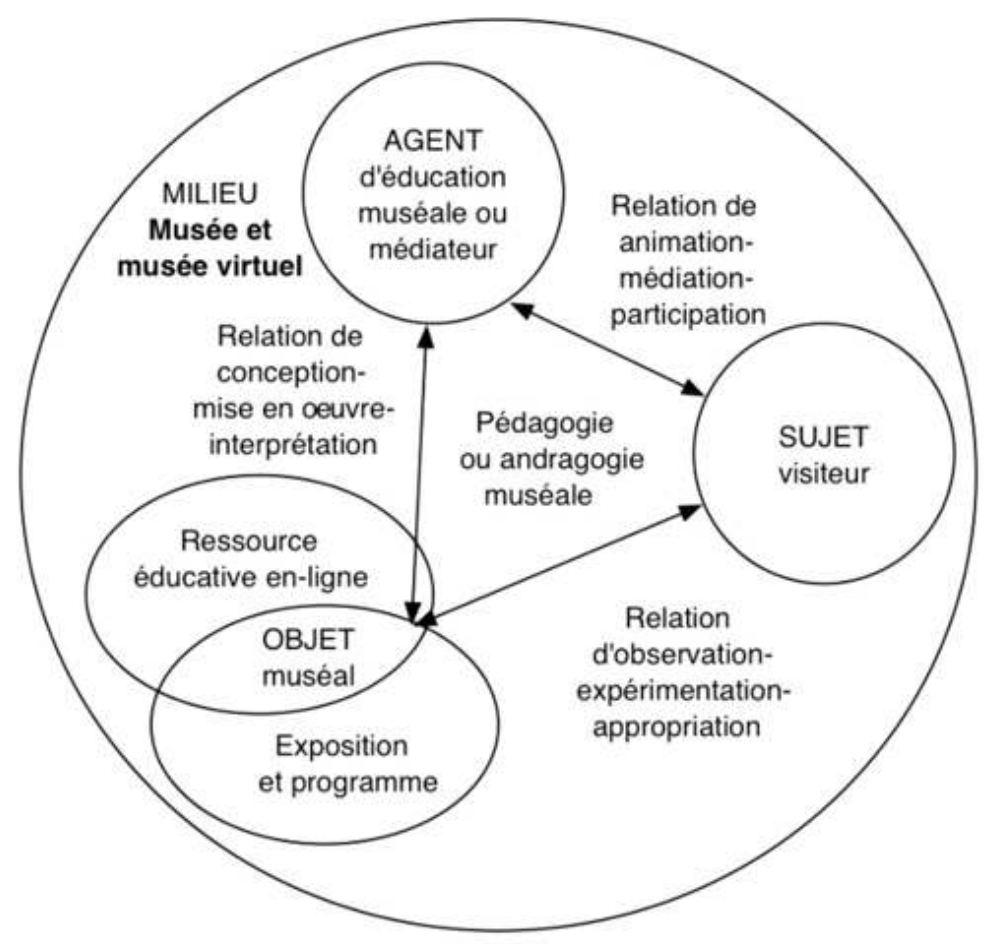

Figure 1 : Modèle d'utilisation du musée et du musée virtuel à des fins éducatives (d'après Paquin et Lemay-Perreault, 2015)

5 Les trois types de relations pour l'étude du web muséal peuvent être décrites comme suit :

- La relation entre l'agent et l'objet : l'agent développe des stratégies pédagogiques et planifie des interventions éducatives. Cette relation comprend le programme éducatif muséal et toutes les ressources éducatives permettant d'atteindre les objectifs d'apprentissage en ligne ou in situ, lesquels se catégorisent en savoirs, savoir-faire et savoir-être.

- La relation entre l'agent et le sujet: l'agent réalise une médiation pour que l'objet soit interprété par le sujet. Cette relation concerne en somme les différentes stratégies pour favoriser l'appropriation de l'objet muséal et de sa signification par le visiteur.

- La relation sujet-objet relève de la démarche du visiteur pour intégrer l'objet d'apprentissage et développer des compétences de visiteur autonome.

Huit sites Internet (et plus spécifiquement leurs sections éducatives) de quatre pays différents (Le Muséum national d'Histoire naturelle, le Louvre, Le British Museum, la National Gallery of London, l'Australian Museum, la National Gallery of Australia, l'American Museum of Natural History et le Moma) ${ }^{1}$ ont été analysés. L'objectif visé était de sélectionner des pays comparables bénéficiant du soutien des politiques publiques et des investissements dans la fonction éducative et dans la société du numérique, dès les années 1990 (Boudjema, 2016). De plus, ces musées jouent un rôle prépondérant sur la scène mondiale dans la recherche, la culture et la diffusion des connaissances. L'internet permet d'établir des liens entre tous les sites ce qui conduit à envisager les dimensions transnationales de la fonction éducative des musées dans la société du numérique. Enfin, ces musées sont engagés dans la préservation de l'environnement et, en ligne, font tous état de cette implication dans l'élaboration et la réalisation d'initiatives afin d'atteindre des objectifs de protection environnementale. 
7 Les travaux récents qui ont lié muséologie et ERE ont permis d'établir une typologie des intentions communicationnelles des producteurs d'expositions. Celle-ci a été développée dans la continuité des apports de Fortin-Debart (2004) sur les différentes approches de l'ERE au musée: interprétative, fondé sur un rapport sensible avec l'environnement; positiviste, basé sur la transmission de connaissances et de comportements; et critique sociale, ancrée sur l'analyse critique des problématiques socio-écologiques. Nous proposons d'analyser et de présenter dans le tableau 1, les ressources éducatives en ligne à l'aulne des objectifs suivants, issus de Zwang (2016) :

- Faire ressentir : l'exposition est basée sur les sens, en particulier la vision, dans le but de provoquer une émotion ;

- Faire connaître : l'exposition renseigne, informe, décrit et expose des constats (statistiques générales) sans entrer dans l'explication sur les causes d'un phénomène ;

- Faire comprendre: la démarche explicative précise les liens de cause à effet d'un phénomène et relève de la volonté du musée à faire accéder le visiteur à des connaissances conceptuelles et complexes ;

- Faire agir : le musée présente les attitudes et les gestes à adopter ou à éviter dans le but que les visiteurs ne les reproduisent ;

- Faire débattre : le musée initie le visiteur au questionnement pour qu'il adopte une opinion à partir d'une réflexion propre et de choix à adopter ;

- Faire valoir : cet objectif relève de la promotion d'actions d'exemplarité du musée ou de ses partenaires, il n'est pas relié à une approche éducative en ERE a priori.

Tableau 1 : Cadre d'analyse des sites web en ERE reliant les approches éducatives en ERE, les objectifs éducatifs des sites internet déduits de leur analyse sémiotique (adapté de Zwang, 2016)

\begin{tabular}{|c|c|c|c|c|c|}
\hline $\begin{array}{c}\text { Approches } \\
\text { educatives en } \\
\text { ERE }\end{array}$ & Interprétative & \multicolumn{2}{|c|}{ Positiviste } & Critique \\
\hline $\begin{array}{c}\text { Objectifs } \\
\text { éducatifs des } \\
\text { sites internet }\end{array}$ & Faire ressentir & Faire agir & $\begin{array}{c}\text { Faire } \\
\text { comprendre }\end{array}$ & Faire connaitre & Faire débattre \\
\hline $\begin{array}{c}\text { Objectifs } \\
\text { centraux }\end{array}$ & $\begin{array}{l}\text { Lien entre la } \\
\text { personne et } \\
\text { l'environnement }\end{array}$ & Les comportements & \multicolumn{1}{|c|}{ Les savoirs } & $\begin{array}{l}\text { Le changement } \\
\text { social }\end{array}$ \\
\hline Basé sur... & $\begin{array}{c}\text { Induction } \\
\text { d'émotions }\end{array}$ & $\begin{array}{c}\text { La présentation } \\
\text { d'actions }\end{array}$ & L'explication & La description & $\begin{array}{c}\text { Le } \\
\text { questionnement }\end{array}$ \\
\hline
\end{tabular}

Les résultats de cette analyse sont exposés dans les lignes ci-dessous.

\section{Une majorité d'approches positivistes centrée sur les savoirs et les comportements}

\section{Les approches basées sur les savoirs favorisées par l'hyperlien}

9 L'approche privilégiée par les sites Web est axée sur les savoirs. L'environnement est objet d'apprentissage. On relève une intention de «faire connaître » et de "faire comprendre ». Cette tendance est principalement observable sur les sites Internet des musées de sciences qui vont déployer plusieurs outils : articles associant textes, images, son, vidéo, interactifs pour montrer des notions similaires et renvoyer à des notions annexes, vidéos, jeux interactifs, parcours thématiques en hyperliens, interviews, questions/réponses, visites virtuelles, objets à la loupe et décryptés par des spécialistes sur le mode interactif. Les fonctions techniques du web reposant sur les liens 
hypertextes s'inscrivent dans une éducation au sujet de l'environnement dans la mesure où elles permettent potentiellement un accroissement des connaissances par un accès à davantage d'informations. La relation est linéaire, le musée met à disposition les informations; le musée en ligne joue un rôle de trait d'union entre le visiteur et l'objet muséal.

10 À titre d'exemple, développons ce qui peut être observé et déduit d'un article mis en ligne sur le site de l'American Museum of Natural History. Dans sa partie «Pre K to Grade $2 »$ (de la moyenne section au cours élémentaire 1) $)^{2}$, le musée présente un article intitulé "What is Biodiversity?» (Qu'est-ce la biodiversité ?). L'intention du site web de placer l'internaute dans une possible acquisition des savoirs est explicitée par la section «Learn and Teach » (apprentissage et enseignement ou Apprendre et Enseigner). Sur la page (figure 2), nous relevons des termes qui renvoient aux savoirs et aux apprentissages visant les savoirs scolaires : "Curriculum» (programme scolaire), avec des propositions en lien avec le programme scolaire. Les étudiants («student»), peuvent apprendre ( (earn ») les concepts basiques relatifs à la biodiversité, ou observer la biodiversité sur le terrain (" field study») dans le but de développer des compétences (" develop the skills»). Le dispositif semble également favoriser l'apprentissage: répétition des concepts, des titres et mots-clés, et proposition de parcours d'apprentissage individualisés via des hyperliens matérialisés sous forme de questions générales (« what is biodiversity?» Qu'est-ce que la biodiversité ?) ou d'adresses à l'internaute (« Not sure how to describe your field site? ? tu ne sais pas décrire ton site? « don't be afraid to ask questions" ne crains pas de poser des questions, "you» (toi)), de verbes d'action («draw!» dessine, "peer into» regarde dans, "head outside» dirige-toi à l'extérieur, etc.). Un guide bibliographique, des ressources éducatives (« educators material » ressources pédagogiques) et des articles complémentaires sont également proposés.

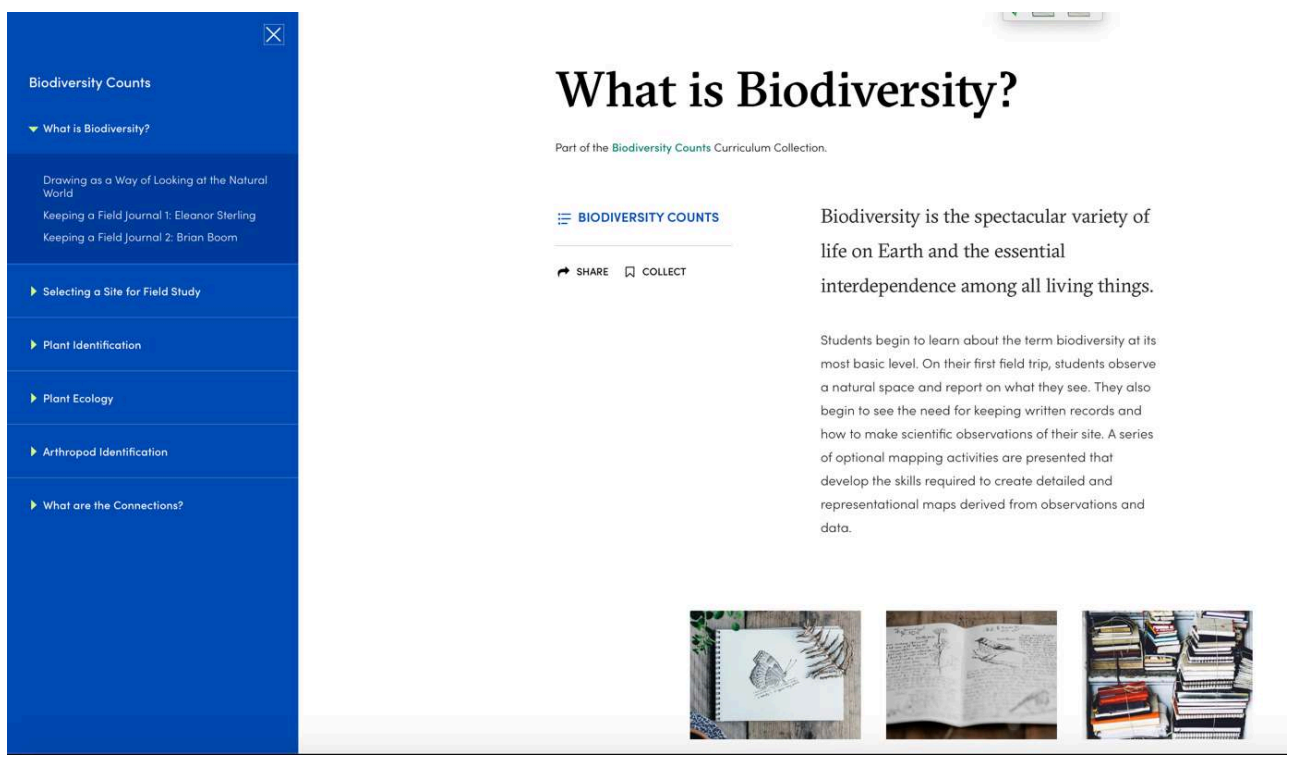

Figure 2 : «What is biodiversity ? " (Qu'est-ce que la biodiversité ?) du site de l'AMNH

11 L'objectif d'acquisition de savoirs est basé sur la découverte ("peer into " regarde attentivement) et l'exploration en ligne de ressources pédagogiques. L'interaction privilégiée entre le musée et l'internaute est celle qui s'adapte aux activités «magistrales» ou interrogatives proposées dans une situation de communication 
individuelle. L'internaute peut, dès lors, bâtir des parcours individualisés d'apprentissage. La structuration des contenus numériques donne une indication sur un possible apprentissage et guide le visiteur à repérer les informations pertinentes pour poursuivre sa navigation. Ici, les contenus sont à appréhender par de la lecture simple et constituent des parcelles et des isolables informationnels.

\section{Les approches basées sur les gestes à adopter}

Dans le cas des gestes et des comportements à adopter, le musée tente de créer des conditions d'un agir sur les attitudes de l'internaute. L'implication du visiteur est visée. Mais cette finalité de former un citoyen écoresponsable, basée à la fois sur sa responsabilité, mais aussi sa culpabilité (Girault et Sauvé, 2008) s'illustre le plus souvent par des prescriptions sur une ligne de conduite individuelle ou collective à adopter. Cette intention de « faire agir » est observable de deux manières.

Premièrement, certains musées comme l'Australian Museum ont recours au texte et à la vidéo, pour inviter l'internaute à réduire son empreinte écologique et à changer ses comportements ${ }^{3}$. Dans la section pédagogique de ce site, un éventail de comportements à adopter en faveur de l'environnement (manger moins de viande et de produits laitiers, acheter des produits bio, acheter des produits locaux, cultiver ses propres produits, etc.) sont présentés de manière injonctive; certains verbes étant mis en exergue en gras : "Think» (Pense), «Eat» (Mange), «Save» (Sauvegarde). L'internaute est ainsi invité à/sommé de réfléchir à ses actions, son alimentation pour préserver l'environnement. La dernière phrase de la page «So Think before you Eat to Save the environment" (Réfléchis avant de manger et Sauvegarde l'environnement) renforce les suggestions "imposées». Le musée ici est prescripteur de comportements, ce qui également notable dans les 14 sections $^{4} \mathrm{du}$ site invitant l'internaute à adopter les gestes pour un développement plus durable ("Sustainability at home » Durabilité au domicile).

Deuxièmement, le musée adopte une stratégie à la fois ludique et prescriptive, mêlant des réalisations créatives et interactives avec des propos scientifiques où il s'agit pour l'internaute d'évaluer son comportement vis-à-vis de l'environnement. Ainsi, sur le site de l'American Museum of Natural History, dans les interactifs sur le changement climatique ${ }^{5}$ l'internaute est invité à découvrir ce qu'il peut faire pour économiser de l'énergie et ralentir le changement climatique. Dans cette page (figure 3), où l'usage de l'impératif, forme neutre sans commandement, est omniprésent : «Explore » (explore), « Find out» (découvre), "Learn» (apprends), "solve » (résous), " explore » (explore), «build» (construis), «think» (pense), «take a closer look» (regarde plus attentivement), l'une des activités, « Be an energy saver » (Sois un économiseur d'énergie), le « Be » semble plus directif (figure 2). Dans un design proche du dessin, par lequel le musée s'adresse aux enfants, l'adresse est faite à l'internaute ("you» toi) et à sa famille pour économiser de l'énergie ("your family" ta famille). Le musée propose alors cinq thématiques (transports, achats, alimentation, domicile, extérieur) avec des gestes et comportements à adopter et dont il s'agit de se rappeler (« reminder»). Pour chaque thématique, l'internaute est convié à vérifier s'il peut réaliser chaque action bénéfique à l'environnement ( $\mathrm{I}$ could do this» (je peux y parvenir), certains hyperliens complètent cette activité pour que l'internaute puisse jouer avec d'autres interactifs ("climate change, energy, fuel, water, recycle» changement climatique, énergie, 
carburant, eau, recyclage, etc.) et apprendre à adopter des gestes pour la préservation de l'environnement grâce à des paroles d'experts.

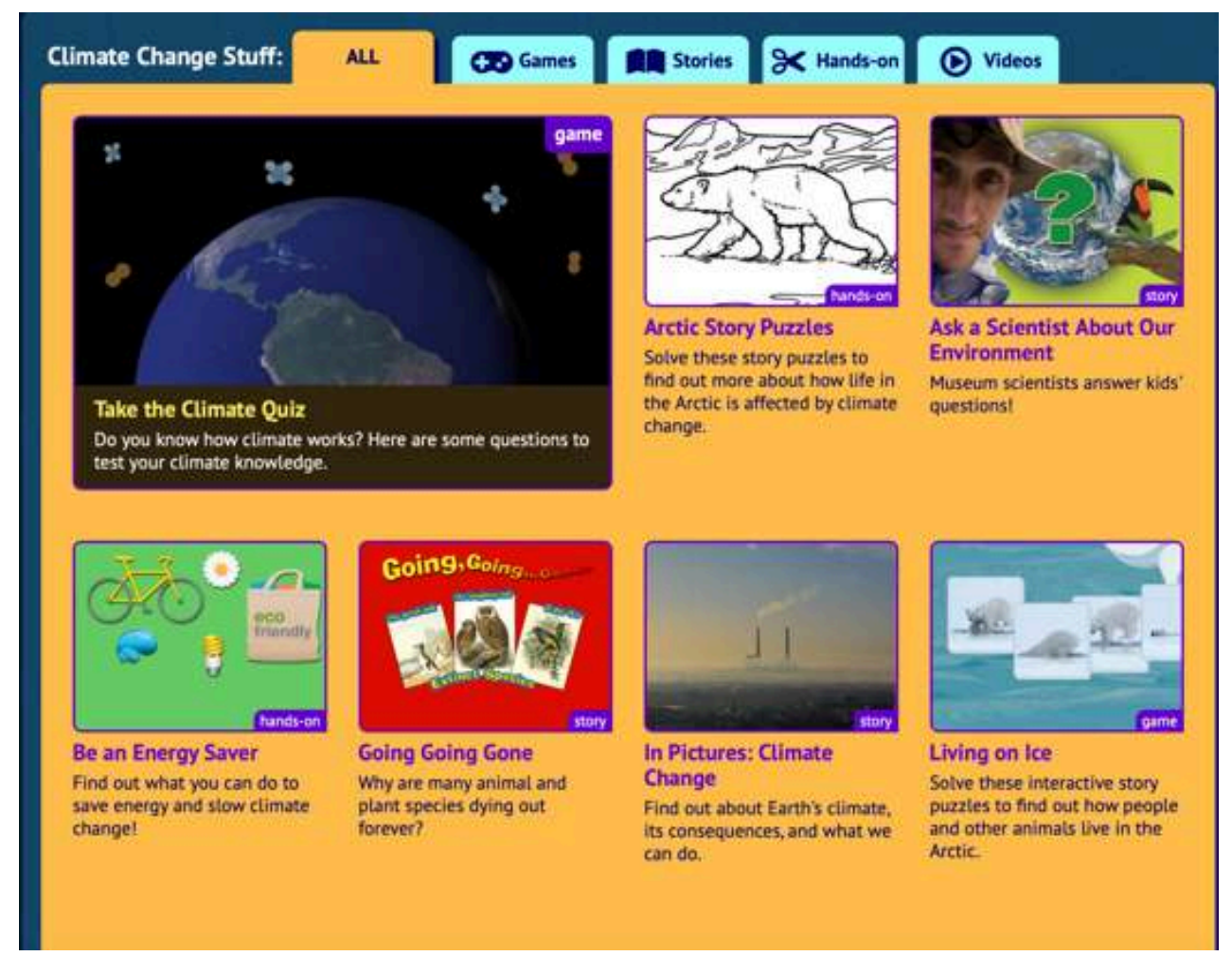

Figure 3 : Les interactifs Climate Change (changement climatique)

Dans ces deux modalités, l'optique est d'intégrer une participation « citoyenne » pour induire la transformation des réalités socio-environnementales qui posent problème. Proches du courant béhavioriste, ces approches envisagent l'apprenant comme un réceptacle d'une réalité environnementale externe, et ne suscite pas l'esprit créatif, sensible et émotionnel de l'apprenant qui reste relativement passif, invité à suivre des directives. Ce qui prime ici est donc que l'internaute devienne capable d'effectuer des actes précis, observables (et évaluables) et d'acquérir des savoirs sans forcément prendre en compte la sensibilité de l'apprenant et du contexte environnemental dans lequel vit celui-ci. On peut dès lors, se demander si, compte tenu de la contrainte imposée par ces injonctions, les motivations et les intérêts se maintiennent suffisamment dans le temps. En effet, cette visée contraste fortement avec les approches critiques qui reposent sur des investigations et un engagement dans l'action collective.

\section{Des approches critiques limitées à des investigations collectives locales}

16 A contrario des approches précédentes, certains musées visent un pouvoir-faire local collectif basé sur des investigations critiques, se rapprochant ainsi du courant de la critique sociale dans lequel l'apprentissage repose sur une participation forte par l'investigation des réalités locales (Fortin-Debart, 2004). Mais cette intentionnalité éducative se limite, dans le peu d'exemples identifiés en ligne, à des campagnes 
collectives de sensibilisation et d'investigation sur un territoire proche de celui des visiteurs.

Ainsi, le Muséum national d'Histoire naturelle, dans sa section "Enseignement et Formation $»^{6}$, développe Vigie-Nature ${ }^{7}$, un programme participatif qui cible les scolaires et vise à assurer un suivi de la biodiversité de proximité. Il offre "des protocoles scientifiques à réaliser avec ses élèves pour assurer des suivis de biodiversité sur l'ensemble du territoire métropolitain $»^{8}$. Si cette approche relève également d'une intentionnalité éducative de construction d'un lien d'appartenance à l'environnement proche, la partie "Biolit " est une forme d'éducation visant des investigations et des choix collectifs. Nous relevons ainsi les verbes tels : "participez (à de nombreuses reprises), aidez-nous à protéger, partagez, à vos observations", etc.). Le musée s'adresse ainsi directement à l'internaute "vos", "vous " et utilise des adverbes d'intensité " plus ", « davantage ", « mieux » permettant à l'institution qui s'exprime de s'impliquer dans la requête. Le pronom "Nous » est particulièrement révélateur dans cette implication et l'aspect relationnel que cherche à établir le musée avec l'internaute. De fait, le protocole est explicité par le «je» (participe, me promène, observe et partage), mais pour un «nous » collectif et communautaire de gestion et de protection de la nature.

Dans la rubrique «Participer $»^{10}$, l'internaute peut choisir entre six actions participatives et aider le musée à réaliser des relevés, des observations, des suivis et des investigations. Pour chaque action, le site énumère les opérations à effectuer pour enregistrer les observations. Le site indique dans des bandeaux le niveau de difficulté des opérations à effectuer. Ces investigations ciblent des choix collectifs afin de diminuer l'impact des activités humaines sur le littoral, transformer les pratiques sociales et résoudre les problèmes environnementaux :

Par vos observations, peut-on parvenir à les identifier, à les connaître davantage pour, à terme, réduire la menace que certaines d'entre elles peuvent représenter? Nous transmettre ce que vous considérez comme une menace sur le littoral, c'est d'une part participer à la sensibilisation sur ces questions, mais c'est également participer ici à la construction d'une méthode pour tenter d'évaluer au mieux les pressions sur cet espace fragile (site Internet: Biolit, Les observateurs du littoral)

Quant à l'Australian Museum (figure 4), il propose en ligne le programme, «Streamwatch» (surveillance des cours d'eau) d'observation des écosystèmes aquatiques locaux $^{11}$, au sein de huit sections pour agir localement sur l'eau et son environnement proche. Le site cible le grand public par une implication "get involved» (soyez/sois impliqué-e-s). Il est ainsi possible pour le citoyen («citizen») de mener des enquêtes (" ecological investigations ») pour identifier les problèmes liés à sa qualité, de trouver des solutions et de décider d'actions locales pour améliorer la qualité de l'eau. La participation recherchée sur le site est signifiée par l'emploi d'images montrant des membres de la communauté ( community») en pleine action d'observation et de relevés scientifiques. Également, tout un champ lexical renvoyant au groupe et au collectif est présent: «community» (communauté), «team» (équipe), community volunteers» (communauté de bénévoles), "hundreds» (des centaines) "groups» (des groupes), volunteer based organisation" (des organisations basées sur le bénévolat), "community conservation», "networks", etc.). L'adhésion au groupe ("citizen water monitoring program» (programme citoyen de surveillance de l'eau), " citizen scientists » citoyens scientifiques) et à la communauté semblent donc centrales 
pour prendre part à ce programme scientifique. Ces actions reposent sur une implication du sujet. Ici, le musée privilégie la cogestion des situations pédagogiques implicantes avec le sujet. Il fournit aux internautes les éléments nécessaires pour mener différentes enquêtes à divers endroits. Il s'agit d'une proposition-cadre au sein de laquelle l'internaute peut choisir ses interventions.

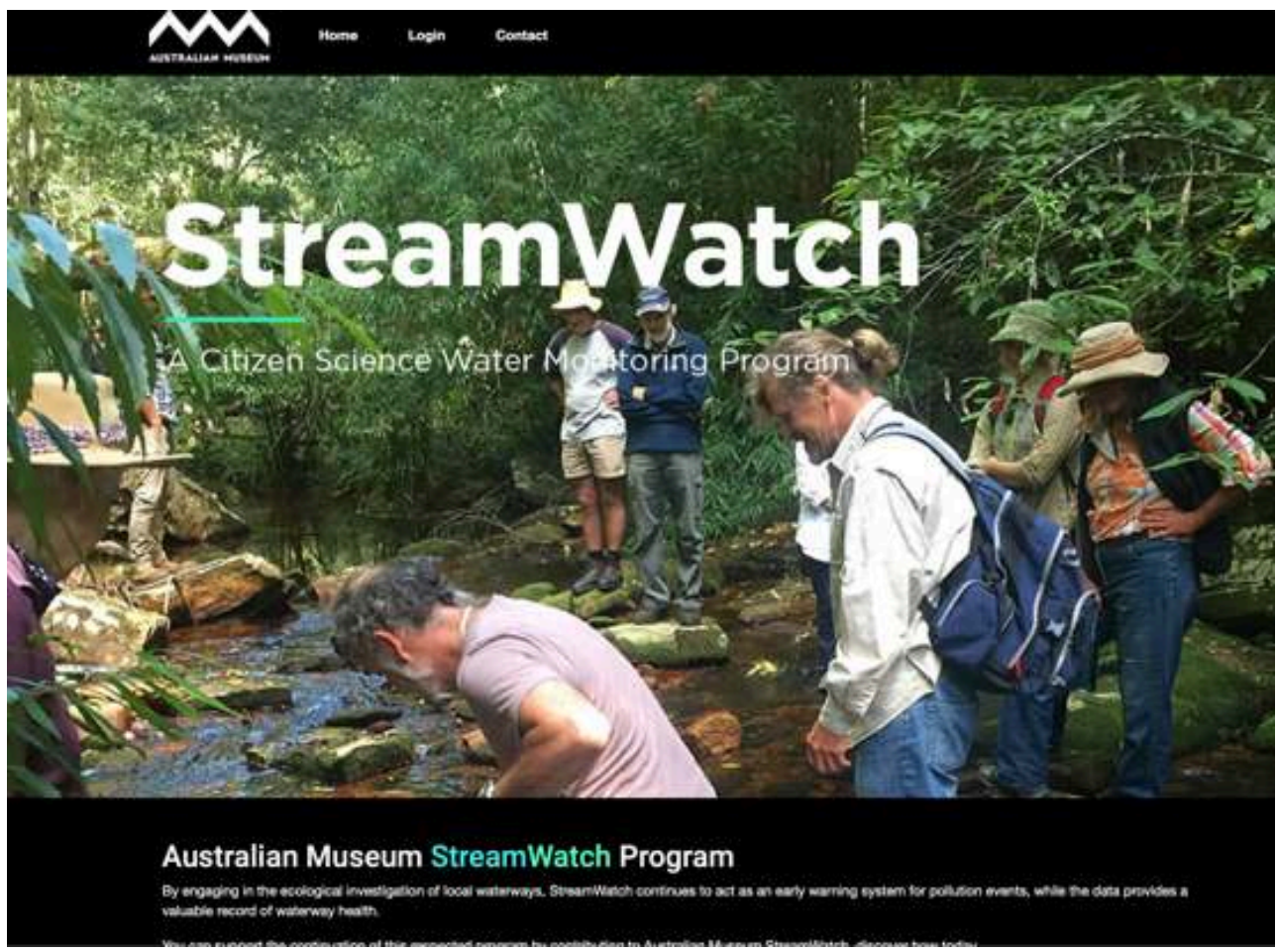

Figure 4 : L'engagement citoyen de Streamwatch

Ces actions reposent sur une participation forte des internautes à mener des enquêtes dans un environnement proche. Cependant, il est nécessaire de souligner que la manière dont le «faire débattre ", qui fait partie intégrante de l'approche de la critique sociale semble totalement absent des sites muséaux étudiés. Le débat est éludé par une focalisation sur des aspects comportementaux ou sur les investigations. En ligne, les finalités éducatives d'une éducation à l'environnement sont donc moins une vision démocratique où l'internaute peut prendre la parole que d'une «nouvelle forme de civisme » (pour reprendre l'expression de Zwang, 2017, p. 120). Plus encore, parce que les musées ciblent les scolaires, on peut supposer que les propos auront tendance à éviter les controverses et s'inscrivent dans un "périmètre d'acceptabilité " (Zwang, 2017, p. 118) ne menant pas, de fait, à une éducation à l'environnement socialement critique.

\section{Des approches interprétatives, entre esthétique et ludification}

\section{" Faire ressentir " par l'approche esthétique}

L'aspect esthétique et le recours à des images esthétisantes traduisent des relations au monde sous le spectre du beau (Zwang, 2017. p. 120). Cette stratégie esthético-affective est employée dans l'optique d'engendrer des émotions pour « favoriser l'empathie avec les autres vivants » (Girault et Sauvé 2008, p. 9) ; inscrivant cette intentionnalité dans 
une forme éducative interprétative (Fortin-Debart, 2004). Il s'agit d'induire des émotions positives pour susciter de l'attendrissement vis-à-vis d'une faune et d'une flore susceptibles de disparaître. À titre d'exemple, sur le site de l'Australian Museum ${ }^{12}$, une page est dédiée à la biodiversité sur laquelle un bandeau (entre le titre et le texte principal) affiche des images touchantes d'animaux dont celle, d'un phoque dans son milieu naturel (prise par le photographe Bernard Spragg) qui regarde dans la direction de l'internaute. L'image crée ainsi ce qu'on appelle en photographie un regard focal fixe (dans la direction du regardant). Cette ligne de regard semble loin d'être neutre du point de vue de la charge émotionnelle puisqu'en général, le regard focal fixé est potentiellement synonyme de danger pour les animaux et que l'homme est le seul mammifère à se regarder durablement dans les yeux (Marcelli, 2009). Or, ici on peut supposer que le musée cherche à établir un échange complice et affectif entre l'animal et l'internaute.

D'autres exemples ont été repérés sur les autres sites web. Ainsi, sur celui du Muséum national d'Histoire naturelle ${ }^{13}$, un article fait état de l'extinction d'une espèce de poisson de récif corallien en mettant en scène un tigre de Sumatra avec divers éléments naturels (faune et flore probablement aussi en voie d'extinction). L'image est en contraste avec le contenu de l'article. En effet, même si ce fauve est en voie d'extinction, on peut se demander pourquoi cette mise en scène - qui place le tigre regardant furtivement droit devant lui - vient introduire un tel article si ce n'est pour susciter une charge émotionnelle auprès des internautes. De même, le site de l'American Museum of Natural History ${ }^{14}$ dans sa partie réservée aux enfants (Ology) met un tigre de Sumatra en scène pour étayer son propos et mettre en place une série de jeux et d'explications autour de l'animal en danger. Cependant, cette fois il s'agit d'un jeune tigre avec toute la symbolique de la fragilité et de la tendresse éventuellement ressenties par les enfants devant leur écran. L'approche esthétique constitue ainsi un ressort usité en ligne par les musées.

\section{"S'immerger » dans l'environnement par le ludique}

Pour favoriser l'empathie pour les espèces menacées, les sites des musées étudiés emploient également une démarche ludique, laquelle dépend particulièrement de la capacité à déclencher auprès de l'internaute, sa curiosité, son intérêt et éventuellement ses questionnements. En ligne, la recherche du plaisir et de l'émotion est poursuivie parallèlement à des fins cognitives.

Par exemple, sur le site de Vigie-Nature, les scolaires sont invités à jouer à la " Compet' à la mangeoire $»^{15}$. Dans cette expérience, les " observateurs-expérimentateurs » sont conviés à compter les oiseaux sur deux mangeoires plateaux identiques. Ces observateurs explorent les choix alimentaires et les interactions entre espèces en fonction de la nourriture disponible et du nombre d'individus sur les mangeoires. L'approche ludique de cette activité invite l'internaute à dépasser les observations scientifiques et à tisser des liens affectifs avec une nature à observer et in fine à respecter. Par le design, soit des dessins ludiques et de belles images d'espèces en gros plan, mais aussi des interpellations directes aux internautes par l'usage de l'impératif ("Contemplez», «bravo vos données sont enregistrées», "découvrez », "téléchargez», etc.) accompagnés de points d'exclamation, le musée stimule l'internaute et l'invite à la participation. 

à la galerie des enfants ${ }^{16}$ (figure 5) et plus particulièrement un interactif qui montre « la nature dans la ville ». L'outil pédagogique se compose de photos, de textes, de vidéos et de jeux et consiste à explorer et à (re)découvrir son propre milieu de vie urbanisé. L'enjeu est de développer un sentiment d'appartenance et de favoriser l'émergence d'une nature à partager. Les jeux « La ville Cache-cache avec la nature dans Paris » et « À la découverte des mystères de la rivière » entendent, à des fins cognitives, susciter des émotions positives vis-à-vis de l'environnement «milieu de vie ». C'est bien l'éveil auprès d'une nature de proximité qui est recherché pour générer un rapport étroit avec les éléments naturels rencontrés dans ce milieu. Les musées font usage de plusieurs stratégies : accompagner la découverte par des « jeux » d'enquête sur le terrain, centrer sur les détails à l'écran (design, humanisation des animaux, gestuelle, couleurs vives, s'adresser à l'enfant "Crois-tu que dans la ville, il n'y a pas de nature? ", "Réfléchis »). À travers ces exemples, le musée est accompagnateur et laisse le sujet organiser ses situations pédagogiques en fonction de ses intérêts. Plusieurs options sont tout de même offertes à l'écran pour le guider dans ses choix. Le musée laisse une part de liberté à l'internaute qui sélectionne en fonction de ses besoins et de ses intérêts dans la cause environnementale proposée à l'écran.

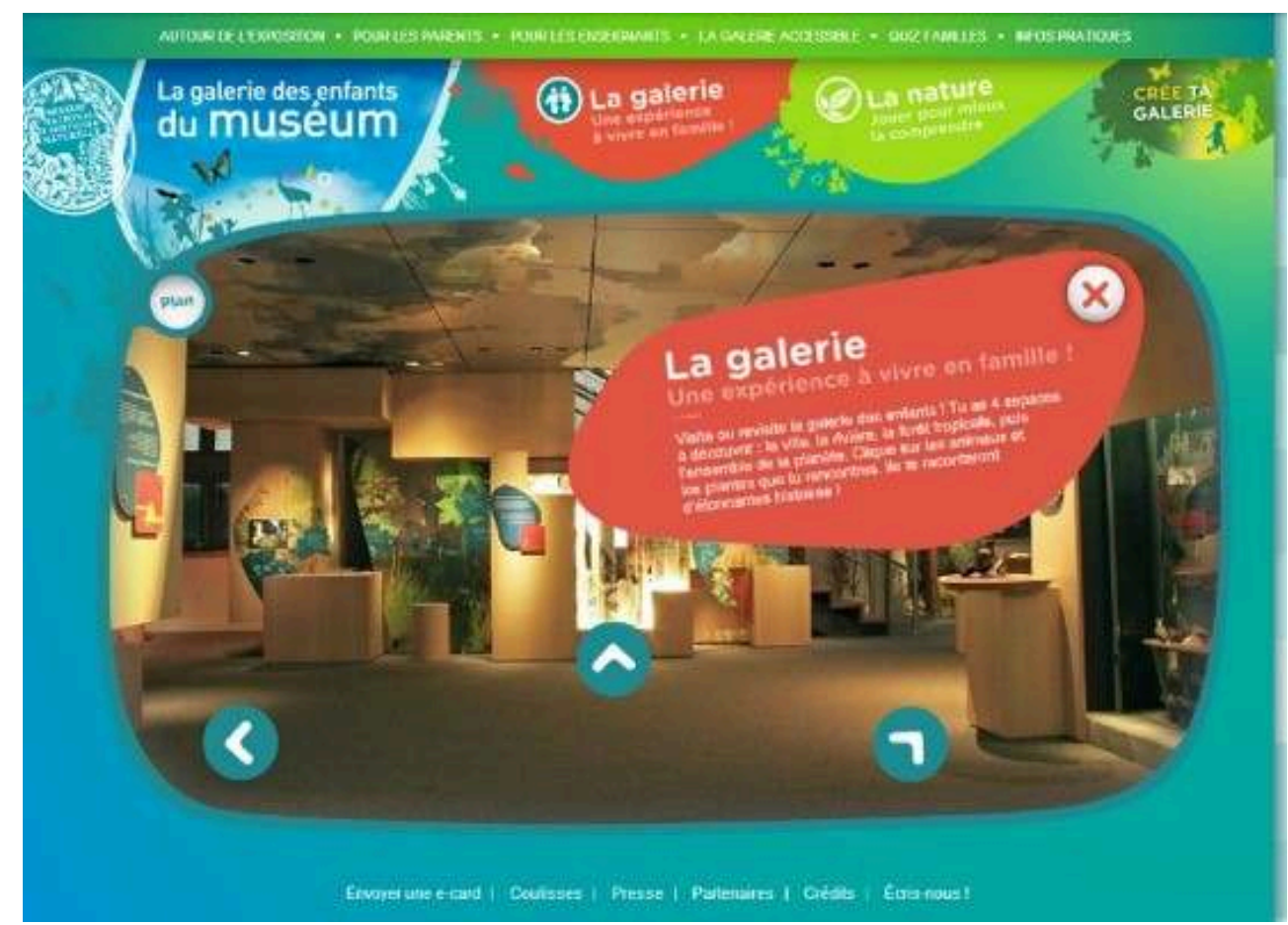

Figure 5 : la Galerie des Enfants et la nature

\section{L'engagement muséal envers l'environnement : un « faire valoir » pour une forme d'éducation à l'environnement?}

Les musées d'art comme les musées de sciences n'hésitent pas à montrer leur engagement dans la préservation de l'environnement. Les divers musées de notre 
corpus mettent ainsi en avant cette implication institutionnelle dans la cause environnementale. Le musée dépasse donc les injonctions de protection de l'environnement en ligne et prône, par ce procédé, une responsabilité qui lui est propre. Ainsi, le site de la National Gallery of London, la rubrique ${ }^{17}$ " SEE ALSO » (regardez également) propose deux thématiques : «Carbon Management plan " (Plan de gestion du carbon), la gestion des émissions de $\mathrm{CO}_{2}$ et "Improving our environment " (améliorons notre environnement) offrant ainsi au musée une opportunité de mettre en avant sa contribution pour limiter son impact environnemental. Le site du British Museum s'attache à présenter l'aspect architectural de ses actions ${ }^{18}$. Le site du Louvre n'hésite pas à afficher son « devoir d'exemplarité ${ }^{19}$ » et entend assurer sa responsabilité environnementale par des actions en faveur des économies d'énergies, de la mobilité durable, des économies de ressources et réduction des déchets, et de la préservation de la biodiversité. Enfin, la National Gallery of Australia ${ }^{20}$ fait état de ses engagements écoresponsables dans l'élaboration des expositions.

In fine, ceci montre comment l'introduction de la thématique de l'environnement change également le rapport des musées au public. En effet, le web est vecteur d'un potentiel « faire valoir » des actions et réflexions menées par les institutions muséales, lequel est aussi employé dans un but de médiation pour encourager le visiteur à s'engager dans des pratiques plus vertueuses vis-à-vis de l'environnement (Arachtingi et Hergault, 2011) via une démarche d'exemplarité. Ainsi le site de l'Australian Museum affiche que le quatrième axe de ses engagements («our committments») est l'éducation des visiteurs et des parties prenantes («educating visitors and stackeholders ${ }^{21} »$ (éduquer les visiteurs et les parties prenantes).

Au-delà des aspects communicationnels, les musées comme d'autres institutions publiques s'engagent dans des démarches collectives du développement durable (Chaumier et Porcedda, 2011). Ces démarches sont apparentes dans toute l'activité muséale : l'architecture et le réaménagement des bâtiments répondent à des normes respectueuses de l'environnement (HQE), les matériels d'exposition font l'objet d'une gestion plus réfléchie et d'un recyclage plus affiché d'une exposition à l'autre (Derouault et Rigogne, 2012). De plus, les musées cherchent la mobilisation de l'ensemble des acteurs de la communauté muséale (personnels, visiteurs, prestataires et un changement de leurs habitudes. On voit donc une double mission : une mission muséographique et une mission de gestionnaire. Gestionnaire de ses ressources indéniablement, mais gestionnaire également dans sa manière de cibler les publics, de proposer des approches multicommunicationnelles (Davallon et coll., 1992).

Face à l'environnement, la dimension institutionnelle du musée est donc repensée car cet enjeu constitue un impact fort en termes muséologique et muséographique. La problématique du rapport musée/environnement dépasse ainsi le niveau «sujet» d'exposition pour aboutir à une logique sociale commune. Le musée «doit» donc s'engager et prendre position dans le débat social sur l'environnement (très polarisé). Il relaie les savoirs scientifiques, mais il « agit » également et le fait valoir, il l'expose en ligne. Il se montre vertueux et affiche sa responsabilisation pour responsabiliser l'internaute (relation avec le public en rétroaction). D'une pédagogie de la connaissance, le musée propose une pédagogie de la responsabilisation. Par conséquent, se pose pour le musée en toile de fond de la responsabilité citoyenne visée, celle de la responsabilité sociale en matière d'environnement. Mais plus encore, grâce à cet engagement social valorisé en ligne, mais aussi à ses activités qui forment le citoyen 
et ses opinions, qui offrent des opportunités d'apprentissage, le musée devient protagoniste du débat social et quitte son statut neutre : « il prend la parole comme un corps constitué de la vie civile » (Davallon et coll., 1992, p. 138).

\section{Conclusion}

La question de l'environnement dans les musées en ligne est une question d'intermédiaires et surtout de relations entre l'agent muséal, l'objet muséal et l'internaute (le sujet). Le dispositif médiatique place donc le public au centre des préoccupations avec cinq types d'objectifs relevés en ligne: faire connaître, faire comprendre ; faire agir ; faire ressentir par l'esthétique et le ludique et faire valoir. À l'interstice de ces intermédiaires, notamment entre l'agent muséal et le sujet internaute, le faire valoir constitue un apport éducatif important. Ce dernier point témoigne de la rencontre de l'environnement avec le musée qui est loin d'être anecdotique dans la mesure où il redéfinit le mode de fonctionnement de l'institution, et ce à double titre. D'abord, il place le musée en tant qu' ' acteur et incubateur de projet» (Chaumier et Porcedda, 2011, p.11) dans ce domaine. Puis, en tant que médiateur de savoirs et de pratiques qui peut déclencher et ambitionner des initiatives citoyennes afin de "relier connaissances du passé et construction de l'avenir» (Chaumier et Porcedda, 2011, p. 11).

31 Le musée n'est pourtant pas une institution qui traite du futur (Davallon et coll., 1992, p. 72). Cependant, le propos éducatif via un certain faire valoir concrétise un lien entre le présent et le futur où le musée se positionne dans des cadres et dispositions aux niveaux social, sociétal, politique et individuel. Pour autant, il est frappant de constater que la muséologie citoyenne qui ambitionne de placer le visiteur en tant que public partenaire et auteur in situ (Boillot, 2011) ne place pas en revanche l'internaute dans une posture du « faire débattre ». Cet objectif est absent des propos muséaux en ligne. Or, les utilisateurs espèrent être en mesure d'interagir socialement à propos du contenu muséal (Dunn et MacDonald, 2009). Les possibilités d'interactions en ligne sont très limitées. De plus, depuis les sections pédagogiques des sites, aucun pont n'est créé vers un réseau social ou un outil de communication où l'internaute peut interpeller le musée et prendre la parole dans un registre du débat. Pourtant, sa prise en compte permettrait d'insuffler un élan citoyen dans les diverses relations relevées. Il s'agit bien de la place du public, au centre des enjeux de la muséologie actuelle. En ligne, malgré les contenus ciblés, la distribution des contenus demeure assez verticale. Pourquoi le musée ne prend-il pas plus en considération l'internaute dans une perspective d'ERE?

Arachtingi, E. et Hergault, M.-C. (2011). Eco-conception, une démarche initiée à Universcience. Dans S. Chaumier et A. Porcedda (dir.), Musées et développement durable (p. XXX). Condé-sur-Noireau : La documentation française.

Baujard, C. (2012). Du musée conservateur au musée virtuel. Systemes d'information management, Volume 18(4), 124-125.

Boillot, F. (2011). L'interaction sociale des musées des sciences de la vie : une mission amnésique ou impossible ? Hermes, La Revue, n 61(3), 188-190.

Boudjema, C. (2016). La fonction éducative des musées dans la société numérique : analyse comparative de l'offre pédagogique en ligne de huit musées nationaux dans 
quatre pays (France, Angleterre, Australie, États-Unis). Thèse inédite. Université Charles de Gaulle - Lille III. Récupéré de https://tel.archives-ouvertes.fr/tel-01405575

Boudjema, C. (2019). L'éducation muséale en ligne : entre savoir réticulaire, coopération et standardisation. Les Cahiers du numérique, vol. 15(1), 119-143.

Chaumier, S. et Porcedda, A. (dir.). (2011). Musées et développement durable. Condésur-Noireau : La documentation française.

Davallon, J. (1999). L'exposition à l'œuvre : stratégies de communication et médiation symbolique. Paris, France : L'Harmattan.

Davallon, J., Grandmont, G. et Schiele, B. (1992). L'environnement entre au musée. Lyon : Presses universitaires de Lyon.

Derouault, S. et Rigogne, A.-H. (2012). Une gestion responsable des expositions temporaires à la Bibliothèque nationale de France. La Lettre de l'OCIM. Musées, Patrimoine et Culture scientifiques et techniques (140), 10-15. https://doi.org/10.4000/ ocim.1035

Dunn, H. et MacDonald, C. (2009). Information culturelle patrimoniale dans un environnement réseauté : comparaison des normes et de l'organisation des connaissances dans les bibliothèques et les musées. Documentation et bibliothèques, 55(4), 159-169. https://doi.org/10.7202/1029180ar

Fortin-Debart, C. (2004). Le partenariat école-musée pour une éducation à l'environnement. Paris : L'Harmattan.

Girault, Y. et Sauvé, L. (2008). L'éducation scientifique, l'éducation à l'environnement et l'éducation pour le développement durable : Croisements, enjeux et mouvances. Dans Y. Girault et L. Sauvé (dir.), Aster - L'éducation à l'environnement ou l'éducation au développement durable (vol. 46, p. 7-30). Lyon : INRP.

Jeanneret, Y. et Labelle, S. (2004). Le texte de réseau comme méta-forme. Dans Actes du colloque Culture, savoirs, supports, le texte n'est-il qu'une métaphore? Thessalonique. Récupéré de https://www.academia.edu/1011494/ Le_texte_de_r \% C3 \% A9seau_comme_m \% C3 \%A9ta-forme

Legendre, R. (dir.). (1993). Dictionnaire actuel de l'éducation (2e éd.). Montréal - Paris : Guérin - Eska.

Marcelli, D. (2009). Se regarder les yeux dans les yeux : un privilège des êtres humains ? Le Carnet PSY, n 139(8), 25-33.

47 Paquin, M. et Lemay-Perreault, R. (2015). Vingt ans de recherche en éducation muséale. Éducation et francophonie, 43(1), 1-12. https://doi.org/10.7202/1030177ar

48 Zwang, A. (2016). La légitimation d'expositions itinérantes pour l'éducation au développement durable : des objectifs de l'École à ceux des producteurs. RDST, 13, 21-49.

49 Zwang, A. (2017). La communication environnementale légitimée pour éduquer au développement durable : de l'attente envers l'autorité éditoriale comme rapport de l'École à la trivialité. Questions de communication (32), 105-124. 


\section{NOTES}

1. Ce corpus initial a été constitué pour une recherche doctorale qui s'attachait à étudier la fonction éducative des musées dans la société du numérique (Boudjema, 2016).

2. https://www.amnh.org/explore/curriculum-collections/biodiversity-counts/what-isbiodiversity, consulté le 23 novembre 2019

3. https://australianmuseum.net.au/blogpost/lifelong-learning/world-environment-day-2013, consulté le 14 octobre 2019

4. https://australianmuseum.net.au/learn/teachers/learning/sustainability/, consulté le 14 octobre 2019

5. https://www.amnh.org/explore/ology/climate-change, consulté le 23 novembre 2019

6. http://www.mnhn.fr/fr/enseignement-formation, consulté le 10 septembre 2019

7. http://www.mnhn.fr/fr/enseignement-formation/actualites/nouveautes-site-vigie-natureecole, consulté le 10 septembre 2019._Voir à ce sujet l'article sur Vigie-Nature dans ce numéro thématique.

8. www.vigienature-ecole.fr, consulté le 11 septembre 2019

9. http://vigienature.mnhn.fr/page/observatoire-du-littoral-biolit, consulté le 11 septembre 2019

10. http://www.biolit.fr/attention-menace, consulté le 11 septembre 2019

11. https://australianmuseum.net.au/streamwatch-get-involved, consulté le 15 octobre 2019

12. https://australianmuseum.net.au/learn/science/biodiversity/, consulté le 15 octobre 2019

13. https://www.mnhn.fr/fr/communiques-presse-dossiers-presse/extinction-especepoisson-recif-corallien-aurait-consequence-extinction-dix-especes-parasites, consulté le 11 septembre 2019

14. https://www.amnh.org/explore/ology/ology-cards/094-sumatran-tiger, consulté le 23 novembre 2019

15. http://vigienature.mnhn.fr/vigie-manip/compet-mangeoire, consulté le 11 septembre 2019

16. http://www.galeriedesenfants.fr/fr/la-nature/la-ville/, consulté le 10 novembre 2017

17. http://www.nationalgallery.org.uk/environmental-conditions, consulté le 8 décembre 2019

18. https://www.britishmuseum.org/about-us/british-museum-story, consulté le 8 décembre 2019

19. https://www.louvre.fr/developpement-durable, consulté le 7 décembre 2019

20. https://nga.gov.au/aboutus/policies/environment.cfm, consulté le 7 décembre 2019

21. https://australianmuseum.net.au/about/organisation/reports/sustainabilityaction-plan/, consulté le 7 décembre 2019 


\section{RÉSUMÉS}

Cet article porte sur une analyse de sites Web de musées en lien avec l'éducation relative à l'environnement et montre les dispositifs pédagogiques sous-jacents. Il met en avant l'implication pédagogique en matière d'éducation muséale en ligne en s'appuyant sur des méthodologies mobilisées en muséologie de l'environnement notamment pour l'analyse des objectifs d'expositions itinérantes environnementales. Il entend ainsi démontrer que le faire valoir constitue une forme potentielle de l'ERE en ligne ce qui n'est pas sans engendrer des questionnements sur le mode de fonctionnement des musées.

This article focuses on an analysis of museum websites related to environmental education and shows the underlying educational devices involved. It highlights the pedagogical involvement in online museum education based on methodologies used in environmental museology, particularly for the analysis of the objectives of itinerant environmental exhibitions dealing with environmental issues. We, thus, intend to demonstrate that emphasizing online environmental museums' actions constitutes a potential form of environmental education, which is not without raising questions about the way museums operate.

\section{INDEX}

Mots-clés : éducation à l'environnement, Web, patrimoine, intentions communicationnelles Keywords : environmental education, Web, heritage, communication intentions

\section{AUTEUR \\ CÉDRIC BOUDJEMA}

Cédric Boudjema est attaché temporaire d'enseignement et de recherche à l'Université Paris 8 au sein du Laboratoire « Paragraphe ». Muséologue, il a été notamment directeur du Fairfield City Museum and Gallery à Fairfield de 2007 à 2011, en Nouvelles Galles du sud en Australie et travaille sur des projets de médiation numérique. Ses recherches portent sur la question de la fonction éducative des musées dans la société du numérique. (Courriel : Cedricboudjema[@]gmail.com) 